\title{
Correction to: Intelligent Antenatal Fetal Monitoring Model Based on Adaptive Neuro-Fuzzy Inference System Through Cardiotocography
}

Xiao-qian Huang, Li Li, Qin-qun Chen, Hang Wei, and Zhi-feng Hao

\author{
Correction to: \\ Chapter "Intelligent Antenatal Fetal Monitoring Model \\ Based on Adaptive Neuro-Fuzzy Inference System \\ Through Cardiotocography" in: B.-y. Cao (ed.), \\ Fuzzy Information and Engineering-2019, Advances \\ in Intelligent Systems and Computing 1094, \\ https://doi.org/10.1007/978-981-15-2459-2_2
}

In the original version of the book, the chapter author name Hang Wei is inadvertently published with spell error "Hag Wei" and it has been corrected now. The chapter and book have been updated with the change. 